\section{PSICOLOGÍA IBEROAMERICANA}

\section{Psicología lberoamericana}

ISSN: 1405-0943

psicología.iberoamericana@uia.mx

Universidad Iberoamericana, Ciudad de

México

México

Hernández Zamora, Zoila E.; Romero Pedraza, Enrique Estrés en Personas Mayores y Estudiantes Universitarios: Un Estudio Comparativo Psicología Iberoamericana, vol. 18, núm. 1, enero-junio, 2010, pp. 56-68

Universidad Iberoamericana, Ciudad de México

Distrito Federal, México

Disponible en: http://www.redalyc.org/articulo.oa?id=133915936007

Cómo citar el artículo

Número completo

- Más información del artículo

Página de la revista en redalyc.org

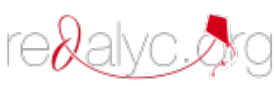

Sistema de Información Científica

Red de Revistas Científicas de América Latina, el Caribe, España y Portugal Proyecto académico sin fines de lucro, desarrollado bajo la iniciativa de acceso abierto 


\title{
Estrés en Personas Mayores y Estudiantes Universitarios: Un Estudio Comparativo
}

\author{
Stress in Aged People and University Students: A Comparative Study
}

\author{
Zoila E. Hernández Zamora \\ Enrique Romero Pedraza * \\ InSTITUTO dE INVESTIGACIONES PSICOLÓGICAS \\ Universidad Veracruzana, MÉxICO
}

\section{RESUMEN}

Se presenta una correlación ente los niveles de estrés de estudiantes universitarios y personas adultas mayores, con el objetivo de determinar cuáles son las principales variables que pueden influir en tales niveles. Se encontró que, de manera estadísticamente significativa, los estudiantes presentan mayor estrés que las personas mayores. Los síntomas que más se presentaron en ellos fueron: cansancio, falta de energía, falta de satisfacción con lo que se hace, intranquilidad, problemas para la toma de decisiones, vida apresurada, falta de seguridad y protección, miedo al futuro y a las responsabilidades, falta de tiempo para sí mismo y plazos de tiempo. Se recomienda tomar las medidas preventivas y correctivas adecuadas para que los niveles de estrés no lleguen a un estado patológico donde resulten nocivos y hasta fatales para la salud de las personas.

Descriptores: Estrés, estresores, estudiantes, adultos mayores, salud.

\section{ABSTRACT}

This article analyzes a correlation between the levels of stress in university students and aged people with the purpose of determining the main variables that can influence such levels. Findings show that students present statistically significant higher levels of stress than aged people. The most common symptoms that students suffered from were: tiredness, lack of energy, lack of satisfaction with what they do, worry, problems in taking decisions, a hurried life, lack of security and protection, fear of the future and of responsibilities, lack of time for themselves and deadlines. We recommend taking preventive and corrective measures in order to avoid that levels of stress become pathological factors which may be harmful and even fatal for people's health.

Key words: Stress, stressors, students, aged people, health.

\section{INTRODUCCIÓN}

En la actualidad, las definiciones del estrés coinciden en calificarlo como "una transacción entre la persona y el ambiente" (Rodríguez, 2001: 60), o una situación resultante de la interpretación y valoración de los acontecimientos que la persona hace. Desde este punto de vista, el estrés "es la condición que resulta cuando las transacciones entre una persona y su ambiente la conducen a percibir una discrepancia (real o no) entre las demandas de la situación y sus recursos biológicos, psicológicos o sociales" (Rodríguez, 2001: 60).

El estrés es el efecto del requerimiento que se le hace a un ser humano. La fuente puede ser externa o

\footnotetext{
* Para correspondencia para cualquiera de los autores dirigirse a: Instituto de Investigaciones Psicológicas de la Universidad Veracruzana, Dr. Luis Castelazo Ayala s/n, Col. Industrial Ánimas, 91190, Xalapa, Ver., México, tel. (228) 8418900 ext. 13208 correo electrónico: zhernandez@uv.mx; eromero@uv.mx.
} 
interna. Por su parte, el requerimiento puede ser positivo o negativo. Según Clegg (2001), se puede decir que no existe vida sin estrés.

Desde la antigüedad existe la creencia popular de que el estrés emocional es causa de enfermedad. Hace sólo unas pocas décadas se convirtió en una idea respetable desde la perspectiva científica, noción que compartían con el mismo entusiasmo tanto los médicos como cualquier otra persona. Se realizaron una serie de estudios que influyeron en forma particular en el convencimiento de una comunidad médica muy escéptica ante la idea de que la mente podía afectar al estado físico (Belsky, 2001).

Es más, el estrés crónico, que resulta de estar de manera continua bajo circunstancias demandantes, puede llegar a ser emocional y físicamente extenuante, con alto riesgo de desarrollar enfermedades y manifestaciones relacionadas con el estrés (Ortega, Ortiz y Coronel, 2007).

El estrés constituye una condición a la que todos los seres humanos se ven expuestos en algún momento o circunstancia de su vida. De acuerdo con Rout y Rout (2002):

Es un proceso complejo en el cual el individuo responde a demandas o situaciones ambientales (estresores) con un patrón de respuestas por parte del organismo que pueden ser fisiológicas, conductuales, cognitivas, emocionales o una combinación de ellas, en el momento de ser interpretadas estas demandas o situaciones como amenazantes. (p. 18)

Para estos autores, los estresores pueden ser eventos vitales, como, por ejemplo, la pérdida de la pareja o de algún familiar significativo, el cambio de escuela, la jubilación, el fracaso escolar, el casamiento o la muerte de un hijo o de alguno de los padres, entre otros, o hasta pequeños incidentes de la vida cotidiana.

Se entiende por evento vital cualquier circunstancia o experiencia en la vida de una persona y/o familia que genera estrés o tensión y cambio. La inadaptación origina cambios en la salud, aparición de enfermedades o agravamiento de las existentes. Precisamente, el periodo del envejecimiento es un proceso irreversible de pérdidas paulatinas y transformaciones a nivel biológico, psicológico y social (Padilla, Gómez \& Gutiérrez, 2005).
En los momentos iniciales del estrés, tales cambios o trastornos son relativamente leves y necesarios ya que permiten al organismo emitir señales que le hacen posible ponerse en guardia y prevenir el desarrollo de problemas más importantes (Rodríguez, Roque \& Molerio, 2002), pero cuando son muy frecuentes se agota la energía, la resistencia y los mecanismos adaptativos de éste dejándolo más vulnerable ante los problemas de salud.

La exposición a situaciones de estrés no es en sí misma algo "malo" o que necesariamente conlleve efectos negativos; sólo cuando las respuestas de estrés -como consecuencia de innumerables estímulos o situaciones ambientales- son excesivamente intensas, frecuentes y duraderas, pueden producir una variedad de trastornos fisiológicos en el organismo y ejercer un efecto psicofisiológico perjudicial que desempeña un papel detonador, regulador o exacerbador de diversos síntomas, así como estados emocionales perjudiciales (Arden, 2002; Pérez, 2002; Rodríguez, et al., 2002).

El estrés puede aumentar cuando la persona es vulnerable, es decir, cuando carece de recursos en una situación de cierta importancia personal. Estos recursos pueden ser físicos o sociales, pero su importancia puede estar determinada por factores psicológicos, como la percepción o la evaluación de la situación (Brannon \& Feist, 2001). Por ejemplo, la artritis en una rodilla puede producir vulnerabilidad física en un joven atleta profesional, pero constituiría un problema común para una persona mayor que no necesita probar sus méritos como deportista.

Son numerosas las variables que intervienen en la aparición del estrés, lo que hace que sus posibles manifestaciones no puedan explicarse mediante un conjunto de relaciones lineales claramente establecidas, sino desde una perspectiva más amplia y menos determinista y generalizable, en la que las variables personales desempeñan un papel fundamental (Peñacoba, 2001).

De acuerdo con el modelo desarrollado por Arnold y Lazarus 1980 (citados por D’Anello, Marcano \& Guerra, 2003) existe una relación estrecha entre satisfacción en la vida y estrés. Específicamente, se plantea que una persona que percibe una demanda proveniente del medio evalúa si ella representa una oportunidad o una amenaza. Si la evaluación es positiva, se produce satisfacción y el proceso se detiene. Si es negativa, se 
produce insatisfacción, un estado de displacer del cual la persona quiere escapar.

El estrés puede aparecer en cualquier etapa de la vida de cualquier ser humano, aunque es de hacer notar que debido al acelerado e imparable envejecimiento poblacional mundial, son cada vez más las personas que arriban a la vejez, la cual, como una etapa más en la evolución del hombre y la mujer, trae aparejados cambios de diversos tipos en el aspecto fisiológico (Blazer, 2000), así como en el orden social, laboral y psicológico, entre otros.

En esta época llega la jubilación, muchas veces sin una preparación adecuada; disminuyen los recursos económicos; hay pérdida de rol social y los consecuentes sentimientos de inutilidad e inconformidad, entre otros, los cuales son causas importantes del desequilibrio emocional de la persona mayor y de los trastornos del estado de ánimo, como la depresión y el estrés (Oliva \& Fernández, 2006).

Sobre el estrés en la vejez existen ideas opuestas, así como acerca del estado emocional en esta etapa vital. Por un lado, se piensa que las personas mayores son pacíficas, reflexivas y satisfechas; por otro, se supone que la vejez es el momento en que se es más vulnerable, más sujeto al estrés y a la depresión debido a la amplia gama de pérdidas que se debe enfrentar. Según Cornachione (2006), en las personas mayores disminuye la capacidad de respuesta al estrés y aumenta la presión arterial. Parece natural que en la vejez las personas se tengan que sentir infelices y sin control, ${ }^{1}$ porque es el momento de la vida en que los refuerzos se tambalean y las pérdidas (o los hechos negativos) son más que las ganancias. Además, los golpes que se sufren en esta etapa de la vida parecen en especial desconcertantes porque son irreversibles, sin que exista en realidad la posibilidad de cambiarlos.

Aunque, contrario a lo expresado, bien pudiera ser que el paso del tiempo -y según como éste sea vivido-, influirá en la adquisición de los recursos que permitirán un manejo del estrés mejor en personas mayores que en jóvenes o en individuos de mediana edad, si se toma en consideración la influencia decisiva del medio específico en el que se desarrolla la persona mayor.

\footnotetext{
${ }^{1}$ El control entendido como la tendencia a sentir, pensar y actuar como alguien importante, y no impotente frente a los avatares de la vida. Ser responsables de lo que les está sucediendo.
}

Los problemas de salud de la juventud, etapa cronológica en la que está incluida la población estudiantil universitaria, son diferentes a los que se presentan en otras etapas de la vida, como la vejez, pues más bien se caracteriza por la carga psicosocial elevada y la baja incidencia de trastornos orgánicos. Los jóvenes son un segmento relativamente sano de la población (Barra, 2004), y cuando reportan mala salud, es por lo regular resultado de accidentes y lesiones producidas por conflictos armados, actos de violencia, uso de drogas o ITS, ${ }^{2}$ principalmente. Los accidentes y las lesiones son las causas principales de la morbilidad, la mortalidad y la discapacidad entre los jóvenes (ONU, 2005).

En esta edad, los factores psicosociales tienen mayor peso que los biológicos en la aparición de estrés, habitualmente generados en los lugares donde viven los jóvenes: centros escolares, deportivos, laborales, de entretenimiento y familiares. Intervenir en los factores de riesgo dentro del proceso educativo imprime a sus actividades un carácter preventivo y de promoción de su salud, más que correctivo, por lo que es una necesidad apremiante aportar información dentro de este campo.

En México, González-Fortaleza (1996) estudió la ocurrencia de estresores familiares, sociales y relativos a la sexualidad entre los jóvenes. Encontró que los varones con estudios de secundaria reportaron un mayor porcentaje de estresores familiares (prohibiciones de sus padres, problemas con éstos por sus bajas calificaciones, hermanos que han interferido en sus vidas), mayor ocurrencia de estresores sociales (problemas con su mejor amigo, sentimientos de ser criticados y ofendidos) y mayor estrés en el área de la sexualidad (prejuicios que les han impedido gozar de su sexualidad, evitar las relaciones sexuales por temor al desprestigio o a contagios, problemas con sus parejas). Además de estudiar la ocurrencia de estresores, González-Fortaleza (1996) estudió los niveles de estrés percibido (evaluación cognitiva) en las esferas familiar, social y sexual de los jóvenes, donde pudo comprobar que los hombres reportaron un nivel significativamente mayor de estrés que las mujeres en el área social y sexual.

Por su parte, Serrano (2003), señala que las jóvenes yucatecas manifiestan mayor estrés por las prohibi-

${ }^{2}$ Infecciones de Transmisión Sexual 
ciones impuestas por sus padres y mayor estrés social que los hombres. Valdés y Pérez (2004) detectaron diferencias por sexo en cuanto a los motivos para suicidarse de jóvenes universitarios, a saber: en los hombres, los problemas existenciales, la pérdida de un ser querido y los problemas económicos; en las mujeres, los problemas familiares y escolares. Como se aprecia en las investigaciones que se realizaron, el papel del estrés es fundamental pues se considera como un primer detonante, a partir del cual el estado emocional resulta afectado (Serrano y Flores, 2005).

En otro estudio que se realizó con estudiantes universitarios, una tercera parte de las situaciones relacionadas con estrés estaban vinculadas con las relaciones personales (Ptacek, Smith y Zonas, 1992). La frecuencia de esta fuente de estrés no debería resultar sorprendente, si se tiene en cuenta el número de relaciones potenciales: los compañeros de trabajo, supervisores, amigos, compañeros sentimentales y relaciones familiares, entre las que se destacan los padres, hijos, cónyuge, tíos, primos, etc. En el caso de estos estudiantes, las relaciones extrafamiliares constituían una fuente de estrés más habitual que las familiares, debido, tal vez, a que sus circunstancias sociales les situaban en un entorno fundamentalmente extrafamiliar (Brannon \& Feist, 2001).

En cuanto a los niveles de estrés en jóvenes y en personas mayores, no existen pruebas de que estas últimas sean más infelices o emocionalmente más descontroladas que los jóvenes. En encuestas realizadas en países tan diferentes como China, Nigeria y Estados Unidos, los ancianos dicen sentirse tan satisfechos con la vida como cualquier otra persona (Diener \& Suh, 1998). Cuando el grupo de investigadores de Powell Lawton (1985) midió la estabilidad emocional de personas mayores que recibían un curso en la universidad, la de sus hijos de mediana edad y la de jóvenes, obtuvieron resultados aún más rotundos. A pesar de tener que enfrentarse a diario con diversas enfermedades crónicas, una cuarta parte de los hombres mayores decía no tener ningún tipo de problemas. Al respecto, se hace necesario realizar más investigaciones en otros grupos de personas mayores de distintas condiciones y profundizar aún más en ellas para comprobar si en realidad existen razones para que la vejez pueda ser un momento de la vida que sorprenda por la ausencia de problemas. Sin embargo, existen personas que luchan con las pérdidas propias de la vejez y resulta interesante que incluso este grupo parece sorprendentemente plácido y relajado. Otro grupo de investigadores comparó las percepciones de estrés entre hombres de mediana edad, ancianos jóvenes y ancianos mayores, y les sorprendió que la mayoría de las personas que se encontraba en el segmento de los mayores, no se quejara de tener problemas (Aldwin, Sutton, Chiara \& Spiro, 1996).

El presente estudio se realizó para constatar si en verdad la tercera edad es una etapa de pérdidas que influyen en la salud emocional de las personas, o bien una época donde se ha aprendido a enfrentar los problemas cotidianos y vitales. Se trata de comparar los niveles de estrés de una población estudiantil universitaria con un grupo de personas mayores, con el objetivo explícito de detectar cuáles son algunas de las variables que influyen en tener un nivel alto, o en su caso, bajo, de estrés.

\section{MÉTODO}

\section{Participantes}

Ciento dos personas adultas mayores (adultos mayores es el término que se aplica a aquellas personas cuyo límite inferior de edad son los 60 años), 39 hombres y 63 mujeres, que representan $38.2 \%$ y $61.8 \%$ de la muestra respectivamente, fueron escogidas de manera voluntaria. Se detectó a aquellos adultos mayores que asisten regularmente al Instituto Nacional para el Adulto Mayor (INAPAM), institución donde se llevó a cabo el estudio y una vez elegidos, se les contactó de manera individual y personal. El que haya mayor cantidad de mujeres se debió a que son muchas más las personas del sexo femenino que asisten a esta Institución que las de sexo masculino.

El tamaño de la muestra se obtuvo a partir de la fórmula para estudios complejos de Rojas (1995) debido a que la población total es menor a 10,000 elementos. Fueron varios los clubes de entre los que se eligieron participantes, a los que se aplicó un cuestionario, a manera de entrevista, compuesto por preguntas abiertas y cerradas, en donde el entrevistador hacía las preguntas y anotaba las respuestas. La instrucción, por tanto, la daba el entrevistador explicándoles los objetivos del estudio y pidiéndoles su colaboración mediante la respuesta a las preguntas que se les realizarían. 
Los integrantes de la muestra tenían un promedio de edad de 69.5 años, con una escolaridad promedio de secundaria; los mayores porcentajes corresponden a personas jubiladas o pensionadas y a amas de casa. El criterio de inclusión fue que tuvieran 60 o más años de edad y desearan participar en el estudio, además de que tuvieran la capacidad cognitiva y sensorial para comprender las preguntas que les realizaría el entrevistador o entrevistadora.

En cuanto a la población universitaria, participaron, seleccionados de manera aleatoria, 119 estudiantes de la Facultad de Psicología de una universidad pública, de diferentes semestres; 29 hombres (24.4\%) y 90 mujeres (75.6\%), con un promedio de edad de 22.1 años. El criterio de selección -elegido por conveniencia-, establecía que pertenecieran a la Facultad de Psicología de la Universidad Veracruzana, ya que esta institución es muy colaborativa en todos los estudios que se realizan, tanto en lo que respecta a su personal como al estudiantado.

\section{Situación o ámbito}

El estudio se llevó a cabo en una estancia diurna dependiente del Desarrollo Integral de la Familia (DIF) estatal exclusiva para el uso de personas mayores de 60 años. Dicha estancia está compuesta por clubes que se agrupan según los intereses de sus integrantes, que se reúnen de una a tres veces a la semana. En promedio asisten 300 personas diariamente a esta Institución.

Con relación a la muestra estudiantil, los cuestionarios fueron aplicados en su facultad, dentro de sus salones de clase, ubicados en la Facultad de Psicología de la Universidad Veracruzana, de la ciudad de Xalapa, Veracruz, institución pública cuya población aproximada es de un total de 500 estudiantes, en su mayoría mujeres (alrededor de 80\%).

\section{Instrumentos}

Cuestionario de datos generales: se aplicó un cuestionario que recababa información sobre edad, sexo, escolaridad, enfermedades, estado de salud percibido, ocupación, estado civil, satisfacción con el apoyo social recibido, estado de ánimo, entre otras cuestiones. Además, mediante dicho instrumento se trataba de identificar los principales estresores percibidos por la población de estudio.
Cuestionario de estrés percibido (PSQ): La escala para valorar el nivel de estrés es una escala tipo Likert que consta de 30 reactivos con tres opciones de respuesta; esta escala fue tomada y modificada de la original de Levenstein, Prantera, Vera, Jarvo, Scribano, Andreali, Luzi et al., (1993), que consta de 20 reactivos y no incluye el componente social de los factores de estrés medioambientales.

En la versión de 20 preguntas de esta escala no se incluyó un componente explícitamente social de los factores de estrés medioambientales. Por ejemplo, en lo que se refiere específicamente a las tensiones interpersonales, sólo uno de los cuatro items se mantuvo ("Siento demasiadas demandas hacia mí"). Lo cual es una posible limitación de la versión breve PSQ. En suma, en la validez de constructo los resultados señalan que el componente psicológico de la percepción de estrés está bien representado por los 20 ítems del PSQ, mientras que el componente social no lo está. Por lo tanto, los estudios que se centran firmemente en cuestiones de estrés social deben preferir el uso de la original de 30 ítems, como es el caso que nos ocupa.

La Escala de Levenstein y colaboradores -"Cuestionario de Estrés Percibido" - (PSQ) refleja, de manera particular, lo que experimentan de manera subjetiva las personas ante determinadas situaciones, y puede ser aplicada a adultos de cualquier sexo, edad y nivel socioeconómico. En ella se presenta una gran variedad de situaciones para que las personas respondan de acuerdo a su interpretación subjetiva. Del análisis factorial resultan siete dimensiones: pensamientos molestos, irritabilidad, falta de alegría, fatiga, preocupaciones, tensiones y demandas (Fliege, Rose, Arck, Levenstein \& Klapp, 2004). En esta investigación, para determinar si las personas se ubicaban en un nivel alto, bajo o medio de estrés, sólo se consideró la puntuación global, o sea, la suma obtenida en las siete dimensiones.

Las características psicométricas demostraron ser favorables. La PSQ muestra una alta correlación con la Escala de Percepción de Estrés de Cohen, el Inventario de Ansiedad (State-Trait-Anxiety Inventory), y la Escala de Depresión (CES-D Depression Scale). En cuanto a la validez externa, en un estudio prospectivo, los autores pudieron predecir los resultados adversos en la salud por medio de valores PSQ. En comparación con 
otras escalas para medir estrés, la PSQ muestra las siguientes ventajas (Levenstein et al., 2000):

1. Desde un punto de vista conceptual, la percepción de estrés se pide lo más directamente posible, sin sugerir las respuestas y sin que el entrevistado se sienta amenazado por los resultados.

2. Evalúa, además de la puntuación general, diferentes facetas de la percepción de estrés que son de interés.

3. Proporciona información, no sólo sobre las respuestas de la persona con relación al estrés, sino también con respecto a la percepción de los estresores externos.

\section{Procedimiento}

Con relación a las personas mayores, se pidió permiso a la autoridad correspondiente para realizar el estudio, a la cual se le explicaron los objetivos y beneficios de la investigación. Luego se obtuvo una muestra aleatoria de las personas que asisten con regularidad a la Institución y, una vez elegidas quienes participarían, se les contactó de manera individual. También a ellas y personalmente se les explicaron los objetivos y beneficios del estudio y se les solicitó su cooperación para responder a la entrevista individual, que estuvo basada en las preguntas del Cuestionario de Estrés Percibido (PSQ) y en el de datos generales. El investigador hacía las preguntas y anotaba las respuestas de los participantes. Por último se llevó a cabo el procesamiento de la información obtenida.

También se solicitó la cooperación voluntaria de los miembros de la población estudiantil que se eligieron de manera aleatoria de la lista del total de alumnos de la Facultad que participarían en el estudio, explicándoles los objetivos de manera grupal. De esta misma manera fueron autoaplicados los instrumentos de medición. En este caso fueron autoaplicados porque, caso contrario de las personas mayores, los estudiantes están más familiarizados con este tipo de pruebas, todos saben leer y escribir y cuentan con la agudeza visual suficiente para poder leer las preguntas. En ambos casos los cuestionarios fueron contestados de forma anónima.

\section{RESULTADOS}

La valoración de los datos se realizó en el SPSS versión 11 de Windows. Se utilizó la chi cuadrada como medida de comparación entre variables, con una significancia de $p<.05$. Se utilizó la prueba de chi cuadrada $\left(\mathrm{X}^{2}\right)$ porque los datos se pueden agrupar en categorías. ${ }^{3} \mathrm{La}$ prueba de chi cuadrada es especialmente útil cuando los datos del estudio de investigación se recopilan en forma de categorías. Algunos ejemplos de dichas categorías son: jóvenes-mayores, estrés bajo-estrés alto, está irritable-no está irritable, se divierte-no se divierte, etcétera.

Tabla 1. Niveles de estrés de personas mayores y estudiantes universitarios

\begin{tabular}{|l|c|c|c|}
\hline Participantes & Estrés alto & $\begin{array}{c}\text { Estrés } \\
\text { medio }\end{array}$ & Estrés bajo \\
\hline Estudiantes & $7.56 \%$ & $55.5 \%$ & $37.0 \%$ \\
\hline $\begin{array}{l}\text { Personas } \\
\text { mayores }\end{array}$ & $2.0 \%$ & $25.5 \%$ & $72.5 \%$ \\
\hline
\end{tabular}

Al comparar nivel bajo con medio entre ambas poblaciones se encontró una diferencia estadísticamente significativa $\left(\mathrm{X}^{2}=24.5\right)$, con una $p<.05$.

Al comparar nivel medio con alto entre ambas poblaciones no se encontró una diferencia estadísticamente significativa $\left(\mathrm{X}^{2}=.50\right), p<.05$.

Al comparar nivel bajo con alto entre ambas poblaciones se encontró una diferencia estadísticamente significativa $\left(\mathrm{X}^{2}=14.1\right), p<.05$.

Significativamente $\left(\mathrm{X}^{2}=27.9\right)$ y con una $p<.05$ tienen un nivel más bajo de estrés las personas mayores comparadas con los estudiantes. De ello se infiere que los estudiantes tienen un mayor nivel de estrés que los adultos mayores, con significancia estadística, lo cual se confirma en la siguiente tabla que compara los resultados de cada una de las preguntas que contenía el instrumento de medición.

\footnotetext{
${ }^{3}$ Una categoría es una división de un esquema lógico de un grupo de personas o una serie de respuestas
} 
Tabla 2. Respuestas comparativas entre estudiantes y adultos mayores correspondientes al instrumento de medición por porcentajes. En todos los casos $\mathrm{p}<.05$

\begin{tabular}{|c|c|c|c|c|c|}
\hline \multirow{2}{*}{ Cuestión } & \multicolumn{2}{|c|}{ Estudiantes } & \multicolumn{2}{|c|}{ Adultos mayores } & \multirow{2}{*}{$X^{2}$} \\
\hline & Sí & No & Sí & No & \\
\hline Se siente descansad@ & 23.5 & 76.5 & 59.8 & 40.2 & 30.0 \\
\hline Siente que le hacen demasiadas peticiones & 16.0 & 84.0 & 15.7 & 84.3 & .003 \\
\hline Con frecuencia está irritable & 8.4 & 91.6 & 10.8 & 89.2 & .36 \\
\hline Siente que tiene demasiadas cosas por hacer & 91.6 & 8.4 & 73.5 & 26.5 & 2.63 \\
\hline Se siente sol@ & 11.8 & 88.2 & 9.8 & 90.2 & .22 \\
\hline Se encuentra sometid@ a conflictos & 44.5 & 55.5 & 37.3 & 62.7 & 1.2 \\
\hline Siente que está haciendo cosas que le gustan & 67.2 & 32.8 & 85.3 & 14.7 & 9.7 \\
\hline Se siente cansad@ & 22.7 & 77.3 & 18.6 & 81.4 & .54 \\
\hline Teme no poder alcanzar sus metas & 7.6 & 92.4 & 20.6 & 79.4 & 7.9 \\
\hline Se siente tranquil@ & 37.0 & 63.0 & 74.5 & 25.5 & 31.1 \\
\hline Tiene que tomar demasiadas decisiones & 79.0 & 21.0 & 44.1 & 55.9 & 28.6 \\
\hline Se siente frustrad@ & 5.9 & 94.1 & 14.7 & 85.3 & 4.77 \\
\hline Se siente Ilen@ de energía & 32.8 & 67.2 & 57.8 & 42.2 & 13.9 \\
\hline Se siente tens@, nervios@ & 16.0 & 84.0 & 8.8 & 91.2 & 23.7 \\
\hline Siente que sus problemas parecen multiplicarse & 6.7 & 93.3 & 9.8 & 90.2 & .69 \\
\hline Siente que tiene prisa & 83.2 & 16.8 & 51.0 & 49.0 & 26.3 \\
\hline Se siente segur@y protegid@ & 47.9 & 52.1 & 83.3 & 16.7 & 30.0 \\
\hline Tiene muchas preocupaciones & 18.5 & 81.5 & 12.7 & 87.3 & 1.4 \\
\hline Está bajo la presión de otr@s & 14.3 & 85.7 & 7.8 & 92.2 & 2.3 \\
\hline Se siente desanimad@ & 5.0 & 95.0 & 8.8 & 91.2 & 1.2 \\
\hline Sedivierte & 47.9 & 32.1 & 60.8 & 39.2 & 3.7 \\
\hline Tiene miedo del futuro & 53.8 & 46.2 & 28.4 & 71.6 & 14.5 \\
\hline Siente que hace las cosas por obligación & 48.7 & 51.3 & 31.4 & 68.6 & 6.9 \\
\hline Se siente criticad@ojuzgad@ & 9.2 & 90.8 & 15.7 & 84.3 & 2.1 \\
\hline Se siente alegre & 64.7 & 35.3 & 74.5 & 25.5 & 2.5 \\
\hline Se siente agotad@ mentalmente & 10.9 & 89.1 & 13.7 & 86.3 & .4 \\
\hline Tiene problemas para relajarse & 15.1 & 84.9 & 18.6 & 81.4 & .5 \\
\hline Se siente agobiad@ por las responsabilidades & 17.6 & 82.4 & 10.8 & 89.2 & 6.6 \\
\hline Tiene tiempo para usted & 37.8 & 62.2 & 72.5 & 27.5 & 26.7 \\
\hline Se siente preocupad@ por los plazos de tiempo & 38.7 & 61.3 & 15.7 & 84.3 & 14.4 \\
\hline
\end{tabular}


La tabla 2 permite observar que a partir de la aplicación de la fórmula estadística se encuentra que significativamente $(p<.05)$ en los adultos mayores, en comparación con los estudiantes:

- Se sienten más descansados

- Sienten más que hacen cosas que realmente les gustan

- Se sienten más tranquilos

- Se sienten más llenos de energía

- Se sienten más seguros y protegidos

- Tienen más tiempo para ellos mismos

- Se sienten más frustrados

- Temen más no poder alcanzar sus metas

También en la tabla 2 se observa que los estudiantes universitarios, en comparación con los adultos mayores y de manera estadísticamente significativa $(p<.05)$, tienen mayores puntuaciones en los aspectos siguientes:

- Sienten más que tienen que tomar demasiadas decisiones

- Se sienten más tensos, más nerviosos

- Sienten más que tienen prisa
- Tienen más miedo del futuro

- Sienten más que tienen que hacer las cosas por obligación

- Se sienten más agobiados por las responsabilidades

- Se sienten más presionados por los plazos de tiempo

En la tabla 3 se presentan algunos datos recogidos a partir de la aplicación del cuestionario general y su respectiva comparación entre estudiantes y adultos mayores.

En la tabla 3 se puede ver que desde un punto de vista estadísticamente significativo, los adultos mayores:

- Tienen más enfermedades que los estudiantes

- Son más los adultos mayores que viven con su pareja que los estudiantes

- Son más los estudiantes que perciben su salud como buena o muy buena que los adultos mayores

- Están más satisfechos los adultos mayores con el apoyo de sus amistades que los estudiantes

- Son más los adultos mayores que se sienten independientes en relación con los estudiantes.

- Son más los adultos mayores que se sienten abrumados por sus problemas que los estudiantes.

Tabla 3. Datos generales comparativos por porcentajes entre estudiantes y adultos mayores. En todos los casos $p<.05$

\begin{tabular}{|l|c|c|c|}
\hline \multicolumn{1}{|c|}{ Variables } & Estudiantes & $\begin{array}{c}\text { Adultos } \\
\text { mayores }\end{array}$ & X $^{2}$ \\
\hline No padece ninguna enfermedad & 76.5 & 28.4 & 63.8 \\
\hline Vive con su pareja & 10.9 & 54.9 & 49.5 \\
\hline Percibe su salud como buena o muy buena & 74.8 & 57.8 & 7.13 \\
\hline Le afectan emocionalmente sus enfermedades & 52.1 & 46.1 & .56 \\
\hline Cree que sus problemas afectan su salud & 56.3 & 57.8 & .03 \\
\hline Está satisfech@ con el apoyo de su familia & 71.4 & 74.5 & .26 \\
\hline Está satisfech@ con el apoyo de sus amistades & 60.5 & 78.4 & 7.26 \\
\hline Se siente independiente & 34.5 & 83.3 & 63.6 \\
\hline Frecuentemente se siente nervios@, triste & 20.2 & 13.7 & 1.56 \\
\hline Se siente abrumad@ por sus problemas & 14.3 & 55.9 & 42.7 \\
\hline
\end{tabular}


En la tabla 4 se presentan los principales estresores de los estudiantes como de los adultos mayores con sus respectivos porcentajes.

\section{DISCUSIÓN}

En oposición a las creencias que afirman que la etapa de la vejez es una etapa de gran estrés debido a la amplia gama de pérdidas que se padece en esta edad, la falta de independencia así como de adaptación a nue- vas situaciones, la pérdida de apoyo social y familiar, entre otras, el presente estudio demuestra que las personas mayores no necesariamente son seres infelices, desprotegidos y hechos a un lado por la sociedad que prefiere los atributos juveniles a los de las personas de edad avanzada. La vejez puede ser una época de ganancias gracias a todas las habilidades y capacidades adquiridas a lo largo de la vida que precisamente contribuyen a enfrentar y/o a compensar las pérdidas que

Tabla 4. Principales estresores de estudiantes y de adultos mayores (en porcentajes)

\begin{tabular}{|l|c|c|}
\hline \multicolumn{1}{|c|}{ Estresores } & Estudiantes & Personas mayores \\
\hline Salud personal & 3.4 & 5.9 \\
\hline Salud de otr@s & 5.0 & 8.8 \\
\hline Problemas económicos & 15.1 & 23.5 \\
\hline Problemas familiares & 23.5 & 30.4 \\
\hline Problemas de pareja & 4.2 & 2.0 \\
\hline Asuntos laborales & 5.9 & 2.0 \\
\hline Incertidumbre (pérdida de control) & 10.1 & 0 \\
\hline Asuntos escolares & 54.6 & 0 \\
\hline Mala administración del tiempo & 16.8 & 0 \\
\hline No tiene ningún estresor (nada los estresa) & 1.7 & 16.7 \\
\hline
\end{tabular}

Nota: En el caso de los estudiantes, el total de los porcentajes excede $100 \%$ debido a que algunos eligieron dos o más alternativas a las preguntas ya que eran abiertas.

Tabla 5. Actividades que realizan para disminuir el estrés tanto estudiantes como adultos mayores en términos de porcentajes

\begin{tabular}{|l|c|c|}
\hline \multicolumn{1}{|c|}{ Actividades para disminuir estrés } & Estudiantes & Adultos mayores \\
\hline Hacer deporte & 12.6 & 25.5 \\
\hline Realizar actividades recreativas & 45.4 & 27.5 \\
\hline Refugiarse en la religión & 0 & 7.8 \\
\hline Refugiarse en sí mism@ & 8.4 & 0 \\
\hline Cuidar su salud & 0 & 5.9 \\
\hline Buscar apoyo social & 12.6 & 6.7 \\
\hline Relajarse o dormir & 39.5 & 13.7 \\
\hline No darle importancia a las cosas & 6.7 & 2.0 \\
\hline Aguantar, no hacer nada & 4.2 & 18.6 \\
\hline Buscar soluciones a sus problemas & 11.8 & 3.9 \\
\hline Llorar & 10.9 & 7.8 \\
\hline Tomar alcohol, fumar o usar fármacos & 4.2 & 4.9 \\
\hline Alteración en la ingesta de comida & 10.1 & 0 \\
\hline Agredir a otros & 5.9 & 0 \\
\hline
\end{tabular}


se sufren, en especial en el último ciclo vital. Como bien puede observarse en los resultados de esta investigación, los adultos mayores presentan niveles de estrés significativamente más bajos $\left(\mathrm{X}^{2}=27.9, p<.05\right)$ que los jóvenes estudiantes, cuyo promedio de edad es de 22 años, situación que puede deberse a los contextos en que se desenvuelven las dos poblaciones, pues en este caso, los primeros tenían el tiempo suficiente y el apoyo para realizar actividades que les gustaban a un lugar especialmente diseñado para ellos, además de ser personas que se consideraban independientes.

En cambio, la situación de los estudiantes es diferente: ellos y ellas están inmersos en ambientes muy distintos a los de las personas mayores, además de tener más y diferentes actividades. La mayor parte de su tiempo la viven en un ambiente escolar que es posible les haga sentirse presionados académicamente y por los rígidos plazos, con poco tiempo para ellos, ya que la mayoría ocupa gran parte del día en actividades escolares, algunos, por satisfacer a los padres o a la sociedad, ya que casi 50\% manifestó hacer las cosas por obligación, no porque les guste hacerlas. Por su parte, los adultos mayores, en su gran mayoría no tenían que cumplir con un horario de trabajo ni de ningún otro tipo, ya que muchos de ellos son jubilados, pensionados y/o amas de casa que ya no tenían hijos en edad de necesitar sus cuidados. Por otra parte, en el cuestionario general se encontró que las personas mayores presentan menor sentido de dependencia que los estudiantes, probablemente porque la gran mayoría de éstos perciben la dependencia como ser sostenidos por sus padres, mientras que los mayores perciben la independencia, aparte de económica (aunque hay algunos que no cuentan con pensión ni ingresos), como la libertad de hacer cosas sin tener que consultarlo o pedirle permiso a otros para hacerlas.

Con relación a lo anterior, cabe recordar lo afirmado por D’Anello, Marcano y Guerra (2003) en cuanto a que existe una relación estrecha entre satisfacción en la vida y estrés.

En cuanto al temor al futuro y a no poder cumplir las metas también hubo una gran diferencia. Mientras que de las personas mayores sólo $28.4 \%$ manifestó temerle al futuro, $53.8 \%$ de los estudiantes pensaba lo mismo (diferencia quizá debida a que los mayores se sienten satisfechos con lo que tienen, o porque sienten que ya no les queda mucho tiempo de vida, o porque disfrutan el presente sin pensar en el futuro).

De manera paradójica, si tenemos en cuenta lo antedicho, los adultos mayores reportaron sentirse más frustrados que los jóvenes, presumiblemente debido a que han vivido más tiempo y en ese tiempo hubo ilusiones, sueños o proyectos que no se cumplieron y que es muy difícil que se lleguen a cumplir en el futuro.

Por otra parte, y también con relación a los resultados, se concluye que no obstante que los jóvenes tienen muchas menos enfermedades orgánicas y percibían su salud en mejores condiciones que la población senescente del estudio, presentan mayores niveles de estrés que las personas mayores, si bien a ninguna de las dos poblaciones les afecta emocionalmente padecer alguna enfermedad.

Los jóvenes, de manera estadísticamente significativa, se encuentran menos satisfechos con el apoyo de sus amistades en comparación con las personas mayores del estudio. En cuanto a los adultos mayores, es probable que, con los años, los lazos amistosos se hayan fortalecido, como lo afirman diversos estudios (Papalia, Wendkos \& Duskin, 2005), y quizá, se tengan menos amistades que cuando se es joven -situación evaluada como no grave-, y si bien, aunque las amistades son menos, los lazos que las unen, además de ser profundos y duraderos, han pasado por muchas pruebas que han sido superadas toda vez que la amistad continúa. Agregado a lo anterior, hay personas mayores sin pareja que sustituyen a ésta por amistades con un fuerte vínculo afectivo.

Ello se explica porque si bien las relaciones personales pueden ser una fuente potencial de estrés, también pueden servir para mitigarlo. En otras palabras, las personas que tienen poca satisfacción con sus relaciones personales presentan un mayor riesgo de contraer enfermedades que aquellas que gozan de una mayor satisfacción con sus relaciones (Berkman \& Syme, Hobfoll \& Vaux, citados en Brannon \& Feist, 2001)

También es notable que sean muchas más las personas mayores que viven en pareja con relación a los estudiantes. En lo que concierne a las relaciones conyugales, en ocasiones éstas pueden constituir una fuente de estrés para ambos componentes de la pareja, lo cual no es el caso en los adultos mayores, puesto que 
el hecho de tener pareja a edades avanzadas más bien constituye un amortiguador para las tensiones y problemas cotidianos, ya que en esta etapa suelen compartirse todo tipo de sentimientos, emociones, preocupaciones, penas y alegrías.

Mientras que los jóvenes manifiestan sentirse más agobiados por las responsabilidades que las personas mayores, éstas últimas se sienten más abrumadas por los problemas. Es presumible que los jóvenes asocien la responsabilidad con las actividades escolares, mientras que los mayores relacionan los problemas con las vicisitudes que se presentan en la vida cotidiana. De acuerdo a Gil-Roales y López (2004), una persona se encuentra bajo estrés cuando tiene que hacer frente a una situación que implica demandas conductuales (responsabilidades, problemas) que no puede satisfacer o le suponen un elevado esfuerzo, lo que se manifestaría por la aparición de una reacción de estrés.

Del estudio también puede inferirse que de acuerdo con la etapa de la vida en que se encuentre la persona, ésta deberá enfrentar estresores diferentes a los que se presentan en otras etapas y contará con diferentes maneras de afrontarlos. Mientras que para los estudiantes su principal estresor son los asuntos escolares, para las personas mayores son los problemas familiares, pues aunque también tales cuestiones son una fuente de estrés para aquellos, lo son aún más los relacionados con el ámbito escolar. Mientras que los jóvenes se enfrentan a sus problemas mediante actividades recreativas o el sueño, las personas mayores lo hacen a través de deportes o actividades recreativas, aunque en menor grado que los primeros. Es factible que también las actividades recreativas que realizan los jóvenes sean distintas a las que llevan a cabo los mayores.

En esta era en la que un gran porcentaje de las personas vive "contrarreloj", muchas de ellas sufren demasiado estrés. Hay dos factores que contribuyen y uno de ellos es la complejidad que se ha añadido al ritmo de la vida. Cuando llega la oleada de adrenalina y las otras hormonas, por lo general no se utilizan los cambios que produce. Es necesario discernir cuándo es necesario luchar o bien, escapar: simplemente, algunas veces hay que sentarse y aceptar las cosas como vienen. Es la combinación del estrés sin una respuesta física y la frecuencia con que la persona se expone a él, de mane- ra casi constante, lo que en verdad causa daño. El cuerpo simplemente no está hecho para una realizar actividades bajo estrés a largo plazo. Sin un escape, el resultado puede ser un incremento del riesgo de algún problema cardiaco u otro tipo de complicación física.

Esta investigación concluye, al igual que otras que se mencionaron al principio, que los adultos mayores son personas que no necesariamente deben padecer un estrés alto, sino que están en condiciones de aprovechar las oportunidades de bienestar, del buen uso de su tiempo libre, así como del apoyo social y familiar con el que cuentan, para mejorar su calidad de vida y lograr una salud que les permita dedicarse a las actividades que deseen o que les gusten.

Porque si bien es cierto que hoy en día se vive a un ritmo en el que las situaciones estresantes parecen multiplicarse, también es una realidad que gran parte de las personas pueden recuperarse de las pérdidas sufridas en etapas anteriores, por ejemplo, perder a la pareja, el empleo o la escuela, y encontrar un compañero o compañera nueva u otro trabajo o escuela o hasta tener las capacidades para afrontar problemas familiares y económicos. Lo importante es tomar las medidas preventivas y correctivas adecuadas para que los niveles de estrés no lleguen a provocar un estado patológico donde resulten nocivos y hasta fatales para la salud de los individuos. 


\section{REFERENCIAS}

Aldwin, C., Sutton, K., Chiara, G. \& Spiro, A. (1996). Age differences in stress, doping, and appraisal: findings from the Normative Aging Study. Journal of Gerontology, 51, 4, 179-188.

Arden, J. (2002). Surviving job stress: How to overcome workday pressures. Franklin Lakes. New York: Career Press Incorporated.

Barra, E. (2004). Apoyo social, estrés y salud. Psicología y Salud, 14, 2, 237-243.

Belsky, J. (2001). Psicología del envejecimiento. Madrid: Paraninfo, S.A.

Blazer, D. (2000). Psiquiatría Geriátrica. En Tratado de Psiquiatría (pp. 46-61). Barcelona: Ediciones Hales/ Udoksky/Masson.

Brannon, L. \& Feist, J. (2001). Psicología de la salud. Madrid: Paraninfo Thomson Learning.

Clegg, B. (2001). Manejo del estrés al instante. México: Ediciones Garnica.

Cornachione, M. (2006). Psicología del desarrollo. Vejez: Aspectos biológicos, psicológicos y sociales. Córdoba: Brujas.

D’Anello, S., Marcano, E. \& Guerra, J. (2003). Estrés ocupacional y satisfacción laboral en médicos del Hospital Universitario de los Andes, Mérida, Venezuela. Revista de la Facultad de Medicina. Universidad de los Andes, 9, 1, 4-9.

Diener, E. y Suh, M. (1998). Subjective wellbeing and age: An international analysis. En K. W. Schaie y E. Lawton (Eds.) Annual Focus on emotion and adult development (pp. 304-324). Nueva York: Springer.

Fliege, H., Rose, M., Arck, P., Levenstein, S. \& Klapp, B. (2004). Validierung des "Perceived Stress Questionaire" (PSQ) an einer deutschen Stichprobe. Diagnostica, 47, 142-152.

Gil Roales, J \& López, F. (2004). Estrés y salud. En Gil, Roales-Nieto, J., Psicología de la salud: aproximación histórica, conceptual y aplicaciones (pp 350-374). Madrid: Pirámide.

González-Fortaleza, C. (1996). Factores protectores y de riesgo de depresión e intentos de suicidio en adolescentes. Tesis inédita de doctorado. México: Universidad Nacional Autónoma de México.

Levenstein S., Prantera C., Varvo V., Scribano M., Berto E., Luzi C., Andreoli A. (1993) Development of the Perceived Stress Questionnaire: a new tool for psychosomatic research. Journal of Psychosomatic Results, 37, 19-32.

Levenstein, S., Prantera, C., Varvo, V., Scribano, M., Andreoli, A., Luzi, C., Arcà, M., Berto, E., Milite, G. \& Marcheggiano, A. (2000). Stress and exacerbation in ulcerative colitis: a prospective study of patients enrolled in remission. Am Journal Gastroenterol, 95, 1213-1220.

Oliva, R. y Fernández, T. (2006). Salud mental y envejecimiento: una experiencia comunitaria de musicoterapia con ancianos deprimidos. Psicología y Salud, 16,1, 93-101.

Organización de las Naciones Unidas (ONU, 2005). La juventud y las Naciones Unidas. Recuperado el 2 de septiembre de 2009 de: http//:www.un.org/youth

Ortega, M., Ortiz, R. \& Coronel, P. (2007) Burnout en médicos y enfermeras y su relación con el constructo personalidad resistente. Psicología y Salud, 17, 1, 5-16.

Padilla, J. Gómez, C. \& Gutiérrez, R. (2005). Eventos vitales del adulto mayor en el área rural del sur del estado de Nuevo León. Revista Salud Pública y Nutrición, 7, 79-85.

Papalia, D., Wendkos, S. \& Duskin, R. (2005). Desarrollo Humano. Cali: McGraw-Hill Interamericana.

Peñacoba, C. (2001). Influencia de la autoestima y el apoyo social en el estrés laboral. Psicología y Salud, $11,1,81-90$.

Pérez, M. (2002). Estrés. Vida o Muerte. México: Instituto Politécnico Nacional.

Powell Lawton, M. (1985). Environment and aging. Chicago: Center for the study of aging.

Ptacek, J., Smith E, \& Zonas, J. (1992). Gender, appraisal and coping: A longitudinal analysis. Journal of Personality, 60, 4, 747-770.

Rodríguez, G., Roque, D. \& Molerio, P. (2002). Estrés laboral, consideraciones sobre sus características y formas de afrontamiento. Psicología Científica. Recuperado el 19 de septiembre de 2009 de: http:// www.psicologiacientifica.com/publicaciones/biblioteca/articulos/ar-gonro01_1.htm.

Rodríguez, J. (2001). Psicología social de la salud. Madrid: Editorial Síntesis.

Rojas, R. (1995). Guía para realizar investigaciones sociales. México: Plaza y Valdés. 
Rout, U. \& Rout, J. (2002). Stress management for primary health care professionals. Hingham, MA: Kluver Academic Publishers.

Serrano, M. (2003). Suicidio, factores protectores y de riesgo en adolescentes de Mérida. Tesis de maestría no publicada. Mérida, Yucatán (México). Universidad Autónoma de Yucatán.
Serrano, M. \& Flores, M. (2005). Estrés, respuestas de afrontamiento e ideación suicida en adolescentes. Psicología y Salud 15, 2, 221-230.

Valdés, M. \& Pérez, B. (2004). La idea de suicidarse en estudiantes universitarios. La Psicología Social en México, Vol. X, 163-168. México: AMEPSO.

Fecha de recepción: octubre 2009

Fecha de publicación: mayo 2010

68 | Psicología Iberoamericana | Zoila E. Hernández Zamora • Enrique Romero Pedraza 